Research Paper

\title{
Structure and Connectivity of Depressive Symptom Networks Corresponding to Early Treatment Response
}

\author{
Eoin McElroy ${ }^{\mathrm{a}}$, Elisa Napoleone ${ }^{\mathrm{b}, \mathrm{c}}$, Miranda Wolpert ${ }^{\mathrm{b}, \mathrm{c}}$, Praveetha Patalay ${ }^{\mathrm{a}, *}$ \\ a Institute of Psychology, Health and Society, University of Liverpool, Liverpool, UK \\ ${ }^{\mathrm{b}}$ Evidence Based Practice Unit, University College London, London, UK \\ c The Anna Freud Centre, London, UK
}

\section{A R T I C L E I N F O}

\section{Article history:}

Received 12 October 2018

Received in revised form 14 February 2019

Accepted 25 February 2019

Available online 14 March 2019

\section{Keywords:}

Depression

Treatment response

Network connectivity

Adolescents

Network analysis

\begin{abstract}
A B S T R A C T
Background: There are suggestions that denser network connectivity (i.e., the strength of associations between individual symptoms) may be a prognostic indicator of poor treatment response in depression. We sought to examine this aspect of depressive symptom networks in the context of early responses to treatment in adolescents. Methods: Routine psychiatric data were obtained for child/adolescent service users who underwent at least three treatment sessions in publicly funded services in England between 2011 and 2015 ( $\mathrm{N}=3017,78 \%$ female; mean age $[S D]=14.43$ years [1.75]). Depressive symptoms were assessed using the Revised Children's Anxiety and Depression Scale at presentation, and again after three treatment sessions. Treatment response was determined using the Reliable Change Index. Network analysis was used to compare the depressive symptom structure and connectivity of sub-samples who, after three treatment sessions had: 1$)$ positively responded $(n=566)$, 2 ) not reliably changed $(n=2277)$, and 3 ) reliably deteriorated $(n=174)$, using matched samples to control for baseline severity.

Findings: Overall connectivity (i.e., the summed total of weighted connections) was significantly weaker for the positive treatment response group at baseline (compared with unchanged and deteriorated groups), however, this group saw the largest increase in connectivity over the course of treatment. With regard to the overall importance of specific symptoms within the networks, fatigue was highest in strength for the unchanged and deteriorated groups, whereas low mood was highest in strength for the improved group.

Interpretation: This study demonstrates that adolescents who respond early to treatment for depression are characterised by symptom networks that are less densely connected initially, yet increase in connectivity over the course of treatment. This may be indicative of 'positive spirals' whereby improvement in one symptom triggers improvements in other symptoms, thereby increasing symptom-symptom associations even as severity decreases.

Funding: The study was supported by the Wellcome Trust grant 204366/Z/16/Z. The funders had no role in the study design, data collection, data analysis, interpretation, or writing of the report.
\end{abstract}

(C) 2019 Published by Elsevier Ltd. This is an open access article under the CC BY-NC-ND license (http://creativecommons.org/licenses/by-nc-nd/4.0/).

\section{Introduction}

Depression is the most prevalent and burdensome form of psychopathology [1-3], with low recovery rates in treatment and high levels of recurrence throughout the lifecourse [4,5].

Despite decades of research into the aetiology, assessment and treatment of depression, we have limited knowledge of the clinical characteristics that differentiate between those who do and do not respond to treatment. It has been suggested that part of this lack of scientific progress may be attributed to our conceptualisation of

\footnotetext{
* Corresponding author.

E-mail address: ppatalay@liverpool.ac.uk (P. Patalay).
}

psychopathology, with categorical diagnostic systems failing to adequately capture the inherent complexity of depression [6].

The network approach is a recently developed alternative that embraces the heterogeneity within psychiatric constructs. Rather than focus on underlying 'disorders', this approach seeks to conceptualise and understand psychopathology as a complex network of locally associated symptoms. Whilst network theory is an increasing area of focus in psychiatric research there is still much debate about how best to use and interpret symptom networks [7-9].

Connectivity (i.e. the strength of the associations that exist between symptoms) is an aspect of the network approach that may be informative in the study of prognosis and treatment response. Indeed, it has been suggested that individuals with strongly associated symptom 


\section{Research in context}

\section{Evidence before this study}

The network approach is a novel conceptualisation of psychopathology symptoms and it has been suggested that this approach might help understand responsiveness to treatment. This approach suggests that psychiatric disorders are the result of causal interplay between individual symptoms, whereby symptoms feed into each other and eventually settle into a mutually reinforcing state. We searched MEDLINE, PsychINFO, and PubMed for studies, that used network analysis to explore group-level differences in those who did and did not respond to treatment for depression, published in English and after 2010 (when network models of psychopathology first emerged) and searched papers that cited the relevant studies found. We identified only two studies that both investigated longer term treatment response and depressive symptom networks. The first study found evidence that network connectivity (i.e. the overall strength of associations between individual symptoms) was higher at baseline for those who had persistent depression after two years compared with those who remitted. As such, it was suggested that more strongly associated symptoms may lead to a poorer prognosis, reflecting maladaptive feedback cycles that are difficult to break. However, a conceptual replication of this study found no such evidence when they examined the symptom connectivity of adolescents who did and did not respond to treatment. We found no previous studies exploring network connectivity and short-term responses to treatment.

\section{Added value of this study}

To the best of our knowledge, we are the first study to explore whether network connectivity is associated with early responses to treatment for depression in adolescence. Second, we utilise a considerably larger clinical sample than the two published studies in this area, and a more reliable approach to determining treatment response. Third, previous studies have only compared networks at baseline, whereas we also explore how these networks change over the course of initial treatment.

\section{Implications of all the available evidence}

Our findings suggest that those with more densely connected symptom networks at baseline are less responsive to treatment in the short-term. Moreover, we found that, for those who demonstrated early positive response to treatment, symptom networks increased in overall connectivity over the course of treatment and changes in individual symptoms indicate uniform improvement across all symptoms. This might suggest 'positive feedback loops', whereby improvements in one symptom may lead to improvements in others.

networks may be less responsive to treatment, regardless of the overall severity of symptoms [10]. Based on this theoretical and methodological approach as conceptualised to-date, at the group-level, this would be reflected in: i) a treatment-resistant group with a symptom network that is densely connected, and ii) a treatment-responsive group with a less densely connected symptom network. The increased connectivity in the treatment resistant group could potentially reflect stronger dynamic interplay and reinforcement amongst symptoms, shared risk factors (e.g. genetic predisposition or environmental factors influencing multiple symptoms simultaneously) or a combination of both.

However, to date, only two studies have examined symptom networks and differences in treatment prognosis in cases of depression.
These studies have both focussed only on baseline connectivity and long-term outcomes, and to date, no research has examined network connectivity as a prognostic factor in of early treatment response. Using data from a prospective study of adult psychiatric patients ( $\mathrm{N}$ $=515$ ), Van Borkulo and colleagues [10] compared the network structures of those with remittent and persistent depression $(\mathrm{N}=515$; assessed at a 2-year follow up), and found that baseline connectivity was stronger in participants with persistent depression. In a conceptual replication, Schweren and associates [11] found no difference in baseline connectivity for adolescents who differed by treatment response $(\mathrm{N}=465$, average months in treatment $=22$ ).

Given the increased visibility of network models in the literature, further research is required to determine whether network connectivity can convey important information regarding prognosis and treatment responsiveness. The present study expands on previous work in several important ways. First, the two previous studies $[10,11]$ focussed their enquiries on groups that differed in their long-term prognostic outcomes. The present study seeks to determine whether group-level connectivity differs in the context of early responses to treatment. Second, the studies of van Borkulo et al. [10], and Schweren et al. [11], relied on relatively small samples, and blunt means of classifying treatment responders/non-responders (e.g. median split at follow-up assessment). In the current study we extend upon the two previous investigations by comparing the overall connectivity and structure of depressive symptom networks in a large clinical sample of children and adolescents, and we use a more robust means of classification, the reliable change index (RCI) [12,13], to define the extent to which overall depressive symptoms changed over three sessions of treatment. The reliable change index permits examination of change in symptoms that is larger than that expected based on measurement error, and hence is a more robust approach to identify groups of individuals that have responded to treatment compared to using median split or raw change scores. Third, there is increased evidence that group-level connectivity can change during the course of illness and in response to treatment [14, 15], yet the two previous studies examined differences in connectivity at baseline only. We expand on previous work by not only examining differences between networks at baseline, but also differences between these networks during treatment, and within-network differences over the course of treatment. Furthermore, we conduct exploratory analyses in which we compare the overall importance of specific symptoms (i.e. symptom centrality) across treatment groups in order to identify symptoms that may be particularly influential in different groups based on their early treatment response.

\section{Methods}

\subsection{Participants}

This study used routinely collected psychiatric assessment data from a national best-practice initiative in the UK between 2011 and 2015. Symptom-level data were collected at 81 Child and Adolescent Mental Health Services (CAMHS) operated by the National Health Service (NHS), local authority and voluntary sector providers [16]. Being a routine clinical database there was considerable heterogeneity in the assessment measures used, treatment strategies implemented, and duration of treatment. Given the focus on depressive symptoms in this study, we started by selecting all individuals aged 8-18 year olds receiving psychological treatment, who had been assessed using the Major Depressive Disorder subscale of the Revised Child Anxiety and Depression Scale (RCADS), and who had complete symptom data available. Based on these criteria, a total of 8238 children underwent routine assessment and at least 1 treatment session, 4687 completed at least 2 assessment/treatment sessions, and 3017 completed at least 3 assessment/treatment sessions. We chose to focus our analysis on the latter group (78\% female; mean age [SD] $=14.43$ [1.75]), as three treatment sessions were judged to be an adequate amount of time to observe 
early responses to treatment whilst also retaining a sufficiently large sample [16]. Compared with those who only underwent one session, our retained sample had a higher proportion of females $\left(X^{2}[1]=\right.$ 148.33, $p<0.01$ ), and were slightly older (mean difference $=$ 8.28 months; $\mathrm{t}[7910]=-14.95, p<0.01$ ). Those who were and were not retained in the sample did not differ in terms of their overall level of depressive symptomatology $(\mathrm{t}[1033]=0.43, p=0.66)$. In our sample, $58 \%$ of individuals were white, $2.4 \%$ were of mixed race, $2.1 \%$ were Asian, $2.1 \%$ were black, $4.4 \%$ were from other ethnic groups, and $31 \%$ did not have their ethnicity recorded. The mean time between first and last third treatment session was 99.11 days $(S D=98.31$; Median $=60.79$; Range $=3-882$ ). The types of psychological treatments received varied considerably for participants, and this information was missing for the majority of the participants (>70\%); therefore, we did not examine effects stratified by treatment type. The data used in this study are service user records and specific ethical permission was not required to conduct this analysis. Children and/or parents who presented for treatment at any of the participating CAHMS providers were asked to sign a consent form indicating that they were happy to have their routine outcome information shared. This form outlined the purposes for collecting such data, the anonymity and data security procedures employed, the voluntary nature of initiative, and their right to withdraw at any time. Approval for this study was granted by the review board of the institution that hosts the data, The Child Outcomes Research Consortium (CORC; https://www.corc.uk.net/), and all data management and confidentiality protocols governing the use of the dataset were followed.

\subsection{Data Statement}

Due to the strict confidentiality protocols surrounding this service user data, the data cannot be shared freely. The correlation matrices used to estimate the networks are available upon request.

\subsection{Measure of Depressive Symptoms}

Symptoms of depression were routinely measured using the Major Depressive Disorder subscale of the Revised Children's Anxiety and Depression Scale (RCADS) [17]. This subscale consists of 10 items scored from Never (0) to Always (3), which are summed to form total severity score (maximum of 30 ).

\subsection{Reliable Change}

We used the RCI $[12,13]$ to divide cases into groups based on the change in scores from the first (T1) to the third (T3) treatment session. The RCI was calculated using the formula in Jacobson \& Truax [12], using the standard deviation and Cronbach's alpha of scores at the first session, resulting in identification of changes that were unlikely to be due to measurement error alone ( $\left.\mathrm{RCI}=\mathrm{x}_{2}-\mathrm{x}_{1} / \mathrm{S}_{\mathrm{diff}}\right)$. After applying this formula to the current dataset, it was calculated that changes in total depression scores of \pm 6.3 were indicative of reliable improvement or deterioration. Three groups were identified using the $\mathrm{RCI}$; i) reliably improved ( $\mathrm{n}=566,78 \%$ female), ii) no reliable change $(\mathrm{n}=2277$, $77 \%$ female), and iii) reliably deteriorated ( $\mathrm{n}=174,81 \%$ female). These groups did not differ in terms of age $(\mathrm{F}(2,3014)=0.84, p>$ $0.05)$, gender $\chi^{2}(2)=1.92, p>0.05$, or length of time in treatment $(\mathrm{F}$ $(2,3014)=2.31, p=0.09)$.

\subsection{Statistical Analysis}

A one-way ANOVA with Tukey post-hoc comparisons was used to compare the overall severity of depressive symptoms (i.e. summed total scores) at T1 and T3. Regularised partial correlation networks were estimated separately at $\mathrm{T} 1$ and $\mathrm{T} 3$ for each of the three groups using the ‘qgraph' package in $\mathrm{R}$ [18]. This package visualises networks as nodes (i.e. points in space that in this case represent symptoms), and edges (i.e. lines linking symptoms). In weighted undirected networks such as these, the edges can be interpreted as partial correlation coefficients, with the thickness of each line reflecting the strength of the association between two symptoms. Each network was visualised using the 'spring' layout, which places strongly connected symptom nodes closer together [19]. To account for the large number of parameters estimated in a typical network, 'qgraph' employs a least absolute shrinkage and selection operator (lasso) to shrink edges and set very small edges to zero, thus reducing the likelihood of false positives [19]. The importance of each individual symptom within the context of the overall network was assessed using two common measures of node centrality; strength (sum of weighted connections) and betweenness (number of times a node lies on the shortest path between two other nodes) [20]. Strength can be considered an indicator of how strongly a symptom is directly associated with other nodes in the network, whereas betweenness highlights how important a symptom is in bridging unconnected symptoms in the network. To determine the extent to which the rank ordering of the edge weights in the networks could be considered reliable, bootstrapped difference tests were performed using the R package 'bootnet' [19]. This procedure takes the observed difference in edge values and constructs bootstrapped 95\% confidence intervals (CIs) around these values. If the $95 \% \mathrm{CI}$ crosses zero, this suggests the edges are not statistically different [19]. The stability of the centrality indices was examined using the case-dropping subset bootstrap method; networks were re-estimated using increasingly smaller subsets of the original sample, and correlations between the original centrality indices and the subset centrality indices were calculated. A small-to-moderate decrease in correlation as participants are removed suggests that the order of centrality is relatively stable/reliable [19].

Networks were compared based on overall connectivity (the summed total of weighted connections, aka global strength) and overall structure using the 'NetworkComparisonTest' (NCT) package [21]. This procedure is carried out in three phases. First, the two networks in question are estimated and the relevant test statistics are calculated. For invariance in overall connectivity, the test statistic is the difference in global-strength (i.e., difference in sum of edge weights of two networks). For structural invariance, the statistic is the largest individual difference in edge strength observed between the two networks. Second, cases are repeatedly and randomly swapped between networks, and these test statistics re-estimated. Third, a reference distribution is created from these test statistics and statistical significance is determined (i.e. whether the observed statistic falls within the 95th percentile for a significance level of 0.05) [10]. Networks were compared using 1000 random permutations. We compared networks across groups at baseline (improved $\mathrm{v}$ unchanged, unchanged $\mathrm{v}$ deteriorated, improved $\mathrm{v}$ deteriorated), and within groups over treatment, meaning a total of 6 network comparisons for conducted. The Benjamini-Hochberg [22] procedure was employed to account for multiple comparisons. Empirical comparisons of the structure and connectivity of symptom networks may be influenced by symptom severity [10], and those who are closer to the extremes of score distributions at baseline are more likely to demonstrate reliable changes in scores over time, i.e. regression towards the mean [23]. Moreover, network comparison procedures may also be influenced by the sample size of the compared groups [10]. As such, to ensure that group-level differences in network structure/connectivity were not driven by baseline severity scores, and to derive equally sized groups, we compared the networks of subgroups that were matched on baseline depressive severity scores. Propensity score matching [24] was used to conduct three matched comparisons at baseline: i) improved group $(n=566)$ v matched controls from the unchanged group $(\mathrm{n}=566)$, ii) deteriorated group $(\mathrm{n}=174) \mathrm{v}$ matched controls from improved group $(n=174)$, and iii) deteriorated group $(\mathrm{n}=174)$ v matched controls from unchanged group. Matching was conducted using the 'nearest neighbour' method in the 'MatchIt' [25] package. 
Table 1

Mean depression scores, standard deviations, and global strength values by response-group.

\begin{tabular}{|c|c|c|c|c|c|}
\hline & $\begin{array}{l}\text { Mean (SD) } \\
\text { T1 }\end{array}$ & $\begin{array}{l}\text { Mean (SD) } \\
\text { T3 }\end{array}$ & $\begin{array}{l}\text { Global Strength Value } \\
\text { T1 }\end{array}$ & $\begin{array}{l}\text { Global Strength Value } \\
\text { T3 }\end{array}$ & $\begin{array}{l}\Delta \text { Global Strength Value } \\
\mathrm{T} 1-\mathrm{T} 3\end{array}$ \\
\hline \multicolumn{6}{|l|}{ Full samples } \\
\hline Reliably Improved $(\mathrm{n}=566)$ & $20.05(4.89)$ & $9.72(5.16)$ & 3.42 & 4.15 & $0.72^{*}$ \\
\hline No Reliable Change $(\mathrm{n}=2277)$ & $17.30(5.87)$ & $16.44(6.34)$ & 4.13 & 4.32 & $0.19^{*}$ \\
\hline Reliably Deteriorated $(\mathrm{n}=174)$ & $12.99(5.16)$ & $22.46(4.8)$ & 3.80 & 3.89 & 0.09 \\
\hline \multicolumn{6}{|l|}{ Matched samples } \\
\hline No Reliable Change Matched to Reliably Improved $(\mathrm{n}=566)$ & $20.05(4.89)$ & - & 3.77 & - & - \\
\hline No Reliable Change Matched to Reliably Deteriorated ( $\mathrm{n}=174$ ) & $12.99(5.16)$ & - & 3.93 & - & - \\
\hline Reliably Improved Matched to Reliably Deteriorated $(\mathrm{n}=174)$ & $12.99(3.52)$ & - & 0.53 & - & - \\
\hline
\end{tabular}

* Difference significant at $p<0.01$ level.

\section{Results}

Mean scores on the RCADS depression subscale are presented for each group in Table 1. At baseline, the reliably improved group had significantly higher scores than the no reliable change group, which in turn scored significantly higher than the deteriorated group $(\mathrm{F}(2,3014)=$ $113.91, p<0.01)$. At T3 the reverse pattern was observed $(\mathrm{F}(2,3014)$ $=400.19, p<0.01$ ). The matching procedure resulted in perfect $1-1$ matches; i.e. participants from the smaller groups were matched with participants from the larger groups who had the same total RCADS depression scale scores at T1 (Table 1). Item-level means at T1 and T3 are presented for each group in Fig. 1 (for standard deviations, see Table S1, available online). There were consistent decreases in means
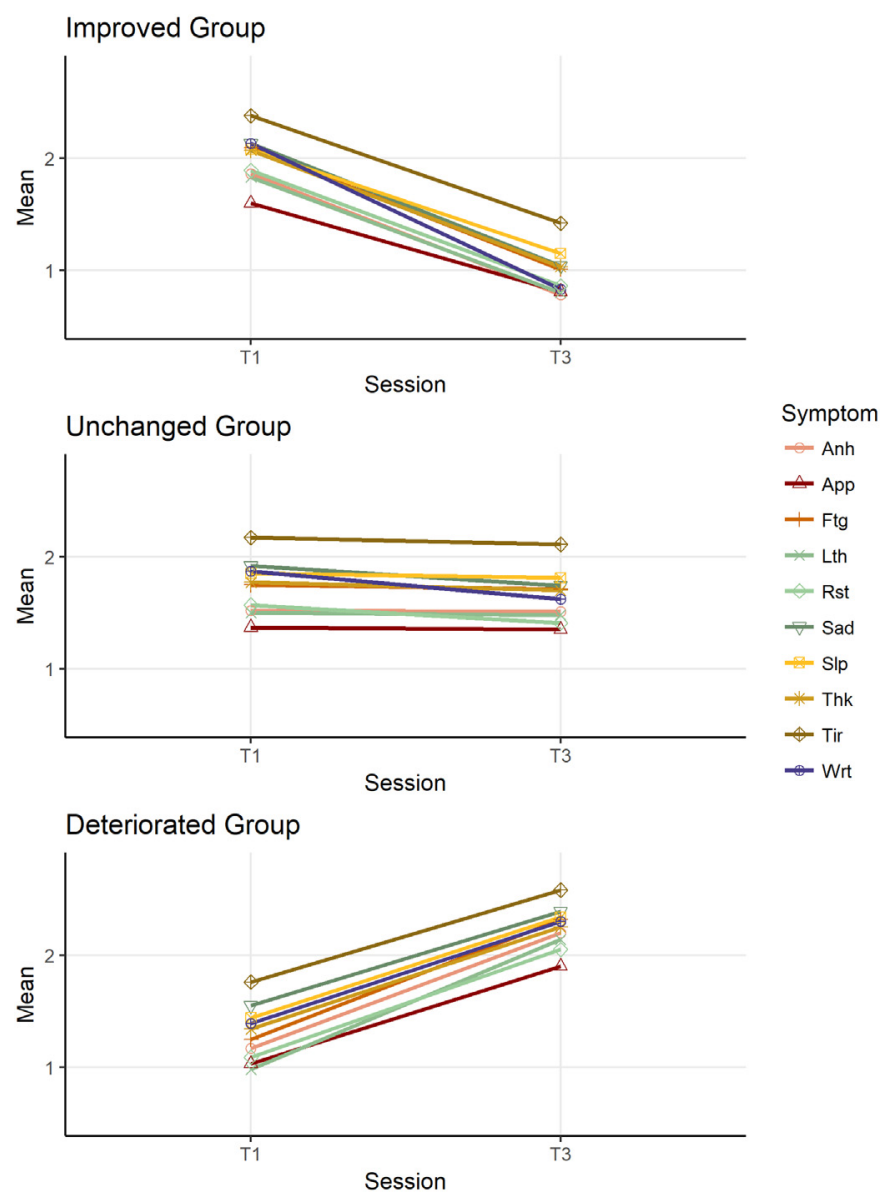

Fig. 1. Mean item scores at baseline and following treatment. Sad = Feels sad or empty; Anh $=$ Anhedonia; Slp = Trouble sleeping; App = Problems with appetite; Ftg = Fatigue; Tir = Tired a lot; Thk = Cannot think clearly; Wrt = Feels worthless; Lth $=$ Lethargy; Rst $=$ Feels restless. across all items for the improved group, and similarly consistent increases for the deteriorated group.

\subsection{Network Comparisons at Baseline}

The network diagrams for the different groups, based on matched samples at T1, are presented in Fig. 2, and global strength values (i.e., overall connectivity) are presented in Table 1.

At baseline, the improved group had a less strongly connected network than matched samples from the unchanged $(n=566, \Delta$ global strength $=0.36, p=0.045)$ and deteriorated $(\mathrm{n}=174, \Delta$ global strength $=3.25, p=0.009$ ) groups, and these remained significant after applying the Benjamini-Hochberg correction. A matched sample from the unchanged group did not differ significantly from the deteriorated group at baseline $(\mathrm{n}=174 ; \Delta$ global strength $=0.13, p=0.62$ ).

\subsection{Network Comparisons Over Treatment}

Connectivity increased for all three groups between T1 and T3, however this change was not significant for the deteriorated group, possibly reflecting lower statistical power (Table 1). In order to further explore where these changes in connectivity were localised, networks were estimated using item-level change scores (i.e. scores at $\mathrm{T} 1$ subtracted from scores at T3), and these networks are presented in the online supplementary materials (Fig. S1). These change score networks demonstrate that the associations between certain symptom pairs changed to a greater degree than others in the improved and unhanged groups, despite the fact that item level changes in severity were fairly uniform (Fig. 1). The NCT did not observe any significant differences in structure within the groups, suggesting that the overall structures of the networks (i.e., which symptoms were most strongly associated with one another) remained broadly consistent across treatment.

\subsection{Centrality: Relevance of Individual Symptoms in the Network}

Centrality indices are presented in Fig. 3. At baseline, fatigue was highest in strength for the unchanged and deteriorated groups, whereas for the improved group, low mood had the highest strength.

Compared with the strength values, betweenness values (number of times a symptom mediates the association between two other symptoms) were more variable across groups. Fatigue had the highest betweenness for the unchanged group at $\mathrm{T} 1$ and $\mathrm{T} 3$, but not for the improved or deteriorated groups.

\subsection{Network Accuracy/Stability}

The bootstrapped difference tests of accuracy and stability are presented in the supplementary material (Figs. S2-S13). The unchanged and improved networks demonstrated excellent accuracy and stability, and the deteriorated group demonstrated moderate accuracy and stability (reflecting smaller $\mathrm{n}$ ). As such the strength of edge weights and rank ordering of centrality can be interpreted with greater confidence 

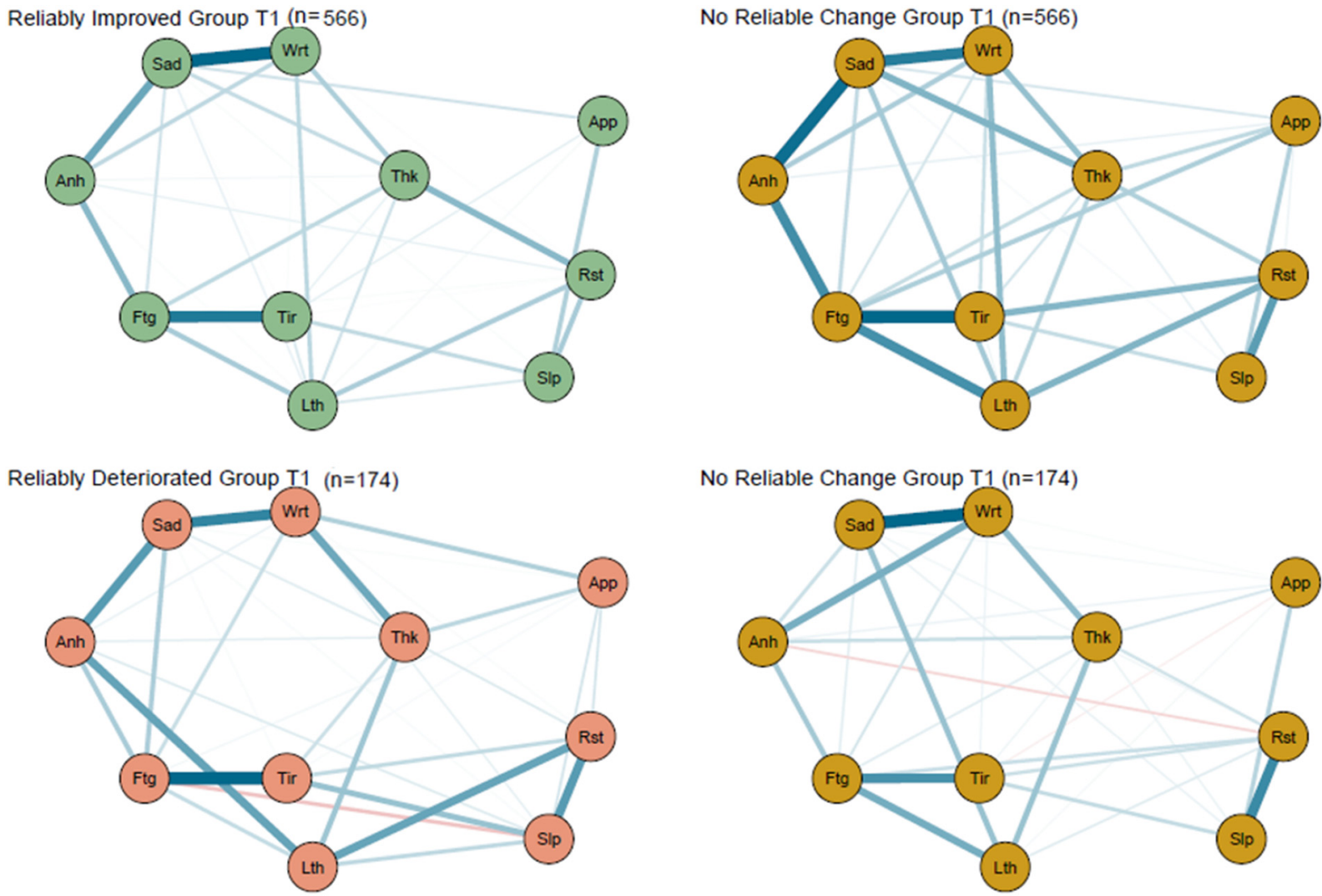

No Reliable Change Group T1 $(n=174)$

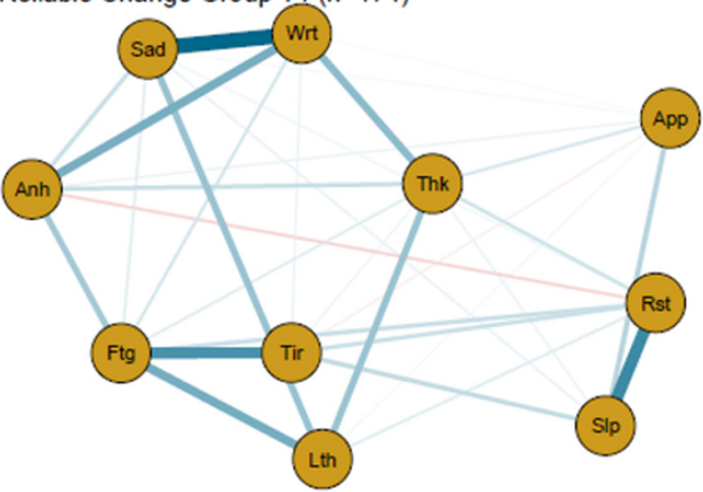

Reliably Improved Group T1 $(\mathrm{n}=174)$
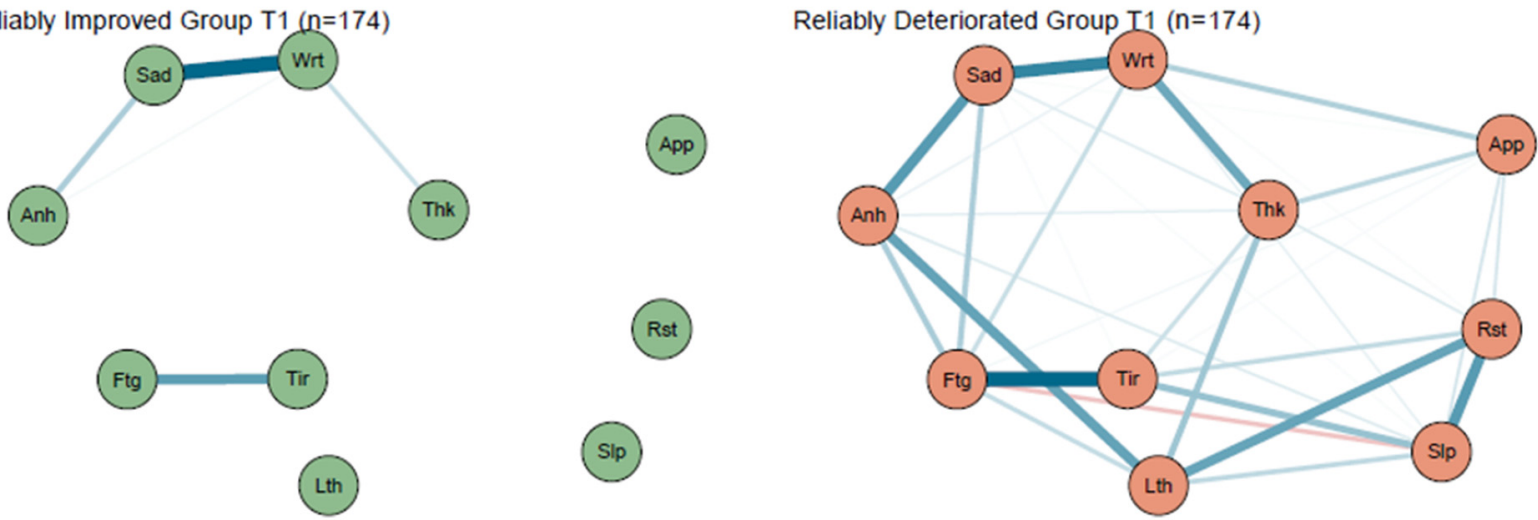

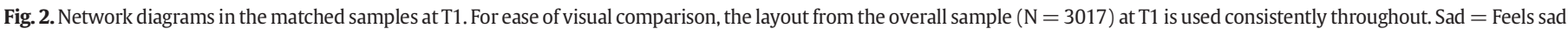

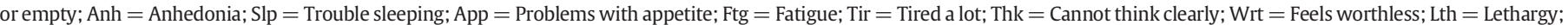
Rst $=$ Feels restless

for the improved and unchanged groups, whereas some caution is advised for the deteriorated group.

\section{Discussion}

This study demonstrates how network models may be used to better our understanding of symptom connectivity in disorder progression and treatment response. We aimed to replicate and extend findings of two previous studies $[10,11]$ utilising a larger clinical sample, a more robust means of categorising those who may be considered responders and non-responders to treatment and examining change in network connectivity and corresponding symptom severity over treatment [12, 13]. We found that independent of symptom severity, the strength of associations (or connectivity) between depressive symptoms is associated with treatment response. Children/adolescents who demonstrated an early positive response to treatment had a less densely connected symptom network at baseline compared with matched samples of children who remained unchanged or deteriorated, replicating one previous study in adults [10]. To further understand the utility of investigating network structure and connectivity in understanding treatment response and progress, we examined changes in network structure alongside treatment sessions and found that network connectivity increased in those with improved symptom severity. It also increased in those with unchanged symptom severity but to a lesser extent. Similar observations of increasing connectivity following treatment have been reported elsewhere $[15,26]$. However, researchers have struggled to interpret this finding [26], as the prevailing narrative has been that higher connectivity is a function of greater severity; i.e., symptoms influence each other and increase overall severity in a negative spiral of reinforcing symptom severity. This has been conceptually illustrated using examples such as insomnia $\rightarrow$ fatigue $\rightarrow$ concentration problems $\rightarrow$ psychomotor problems [27]. Such 'negative spirals' have been framed within the clinical staging model [28], whereby disorders progress from subclinical symptoms to chronic and severe pathology, with 

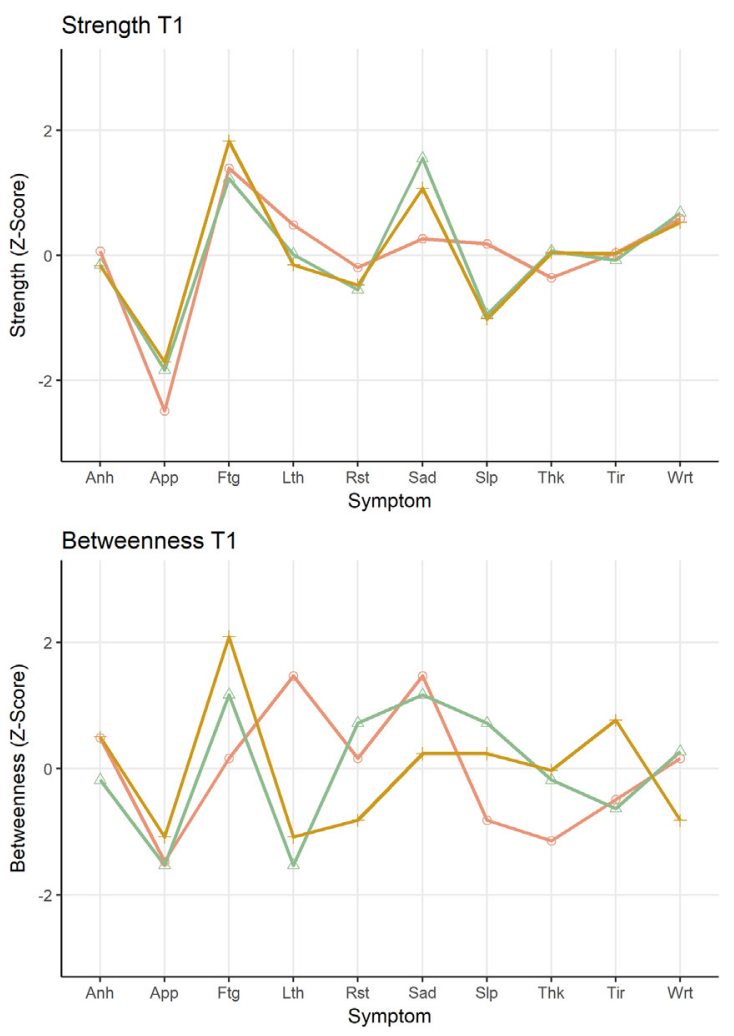
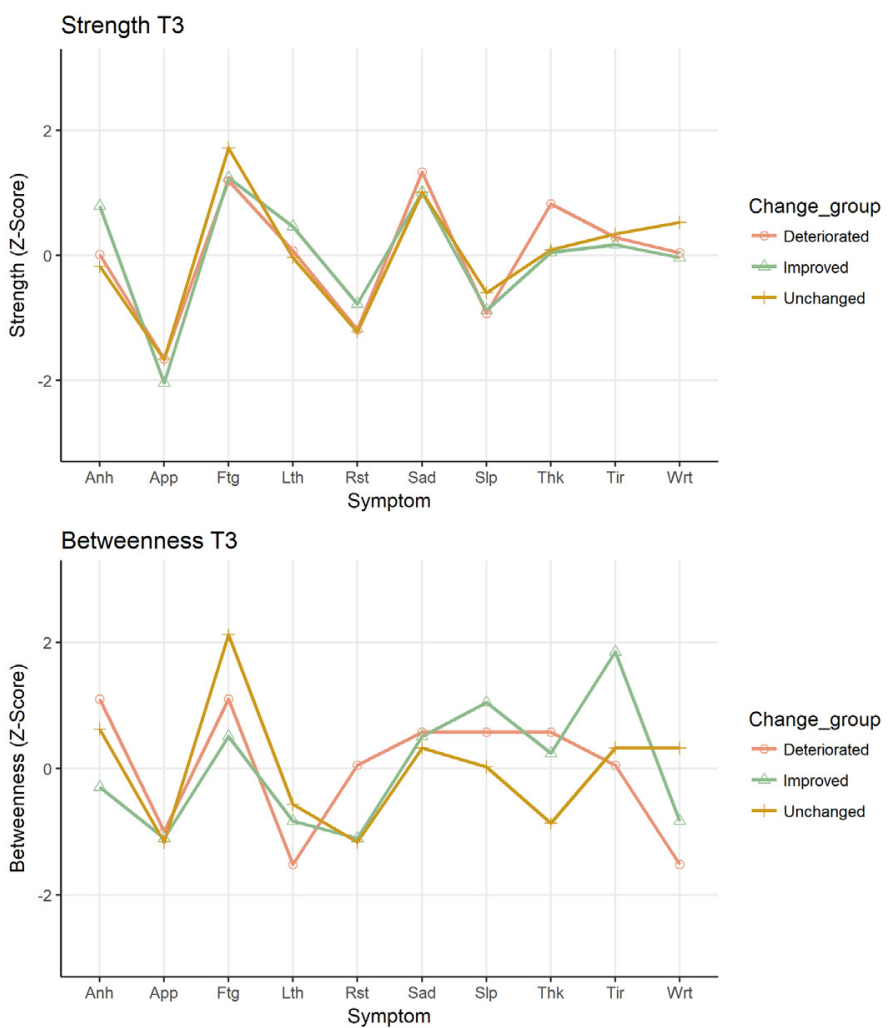

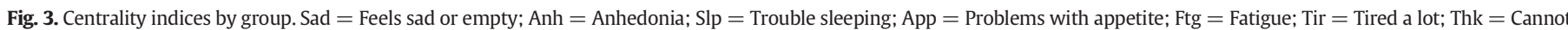
think clearly; Wrt $=$ Feels worthless; Lth $=$ Lethargy; Rst $=$ Feels restless.

stronger associations between symptoms facilitating this progression [29]. In light of this framing, a possible explanation for our observed findings of increased connectivity alongside decreased symptom severity is that the reverse could plausibly occur; i.e., 'positive spirals' whereby improvement in one symptom leads to an improvement in another symptom (e.g. decreased sleep problems $\rightarrow$ increased energy levels and motivation). With this hypothesised explanation although overall severity in symptoms would decrease, the associations between symptoms might increase in the treatment-responsive population. In the present study, the higher increased connectivity observed in the reliably improved group could be interpreted as supportive of this idea, and may explain why an increase in connectivity between symptoms can be found even as overall severity decreases $[15,26]$.

Another possible explanation that has been proposed to explain the higher increased connectivity observed in the group responsive to treatment is a change in the interpretation of items and how they relate to one another due to the therapeutic effects of psychological treatment (response shift bias) [15]. Furthermore, it has been suggested that group-level differences in connectivity may be explained by differences in item variances [30]. Indeed, recent studies have found that the variance of symptoms increased as the severity of depressive symptoms decreased in response to treatment $[14,15]$. However, in the present study, we found that, with the exception of one symptom ('Feels tired'), items in the improved group (which saw the only significant increase in connectivity) decreased slightly in variance over the course of treatment. Thus, this explanation does not explain the increased connectivity observed in the improved group and this cannot be attributed to increased variance in these data.

We further explored changes in network structure and connectivity over treatment by estimating networks based on change scores. The change network for the improved group was sparse with few connections. This demonstrates that, although the mean-level severity of symptoms decreased in a fairly uniform way over treatment, changes in the associations between items were not consistent. This lends support to the possibility of positive spirals of symptom improvement that might occur in response to treatment in some individuals leading to improvement in overall levels of depression. As such, this highlights that it is crucial to interpret changes in network structure together with changes in symptom-level severity. In order to accurately capture such symptom improvement or deterioration spirals, further research employing designs that collect repeated measures over short intervals (i.e., experience sampling method) is required [31].

The reliably deteriorated group identified in the present study did not see an increase in overall connectivity over treatment which does not support the expectation that increased connectivity might help explain increased overall severity. It is important to note the smaller sample size of this group, and future research with larger samples would benefit from further investigating the characteristics of those who deteriorate during treatment. As for the unchanged group, a small increase in overall connectivity was observed, possibly reflecting shifts towards recovery or deterioration that was occurring at a slower rate, and as such had not (yet) resulted in meaningful clinical change in symptom severity.

Along with overall connectivity, the present study explored differences in the importance of individual symptoms in the various treatment response groups, focussing on the strength centrality (i.e. how strongly a symptom was directly associated with all other symptoms) [19]. Across all three treatment response groups, there was consistency in the relative strength values of different symptoms. Fatigue was particularly high in strength for all three groups at both time points. This finding is in line with previous studies, using adult samples, which have highlighted fatigue and lethargy/psychomotor retardation as highly central symptoms in cases of depression [10,32,33]. Investigating the rank ordering of centrality values, we observe that the fatigue symptom was highest in strength for both the unchanged and deteriorated group, whereas for the improved group the symptom 'sad/low mood' had the highest strength. Tentatively, this suggests that fatigue might be a core symptom amongst those who do not respond to treatment. 
This not only offers a plausible mechanism for the persistence of symptoms over treatment (i.e. fatigue/lethargy impeding efforts to successfully implement treatment strategies), but also suggests an area for direct intervention, for instance with treatments aimed specifically at reducing fatigue, particularly in cases of chronic depression. To the best of our knowledge, research has not yet explicitly examined the effect of intervening on highly central symptoms (compared with peripheral symptoms, or construct-level interventions), however this could be a promising area for further enquiry.

The main strengths of the present study were the large clinical sample, the use of a robust means of classifying individuals based on their response to treatment, and the use of a well-validated clinical measure of depressive symptomatology. As a result of using reliable change in symptom severity to determine treatment responsiveness, a small proportion of individuals were identified as having reliably deteriorated during the course of treatment. This group is expected to exist in usual treatment settings [16], but has not been studied to-date in the context of psychopathological networks $[10,11]$. It is important to note that this deteriorated group had a smaller sample size, meaning less reliable information about their symptom networks is available. By using propensity score matching to match groups on baseline symptom severity, we can be confident that these findings are not just reflecting differences between groups in baseline severity. With regard to limitations, first, due to considerable missingness and heterogeneity in treatment data, we lacked the data to factor into our analysis the type of treatment undergone. It also remains possible that responsiveness may have differed as a function of treatment type [14]. Second, there were no data available on any co-administered psychopharmacological treatments, and recent evidence suggests such treatments can alter the structure of symptom networks [14]. Third, given the study used real-world routine psychiatric data, the length of treatment varied for each individual. Fourth, the major depression subscale of the RCADS contains one item ('Appetite problems') which likely encompasses opposing symptomatology (i.e., eating too much/not enough), which makes it impossible to disentangle unique associations for these symptoms. Fifth, although co-morbidity is common and studying the symptom networks of any co-morbid conditions might be informative, routinely collected symptom data on any potential co-morbidities were unavailable. That is because in routine care it is unfeasible and uncommon to collect several outcome measures routinely from each service user. Finally, it must be noted that the networks reported in the present study were crosssectional/between-participant networks. Whether findings from such networks can be generalised to the level of the individual remains to be determined [34].

In conclusion, using a large clinical sample we demonstrated that individuals who were the most responsive to treatment over a small number of sessions had a less densely connected symptom network at baseline, and this symptom network increased in connectivity over the course of treatment. Fatigue was higher in centrality for those who did not respond positively to treatment (compared with those who did), suggesting it might play a key role in determining treatment response. For those who reliably improved over the first three sessions, increases in connectivity may be the result of positive spirals, whereby improvements in one symptom influences other symptoms in the network, thus increasing symptom connectivity over treatment course. This finding suggests that treatment response may not simply be determined by how severe symptoms are throughout treatment, but also how these symptoms are associated and how these associations change over time.

\section{Conflict of Interest}

Dr. McElroy reports grants from Wellcome Trust, during the conduct of the study; grant 204366/Z/16/Z. Ms. Napoleone has nothing to disclose. Prof. Wolpert has nothing to disclose. Dr. Patalay reports grants from Wellcome Trust during the conduct of the study (grant 204,366/
Z/16/Z) and grants from ESRC, Big Lottery, Department for Education (England), outside the submitted work.

\section{Acknowledgements}

The authors would like to thank all partnerships that took part in the Children and Young People's Improving Access to Psychological Therapies (CYP IAPT) service transformation programme between 2011 and 2015 for providing the data presented here. The authors would also like to thank members of the Child Outcomes Research Consortium (CORC), its committee at the time of writing: Miranda Wolpert, Ashley Wyatt, Mick Atkinson, Kate Martin, Ann York, Alan Ovendon, Duncan Law, Julie Elliot, Isobel Fleming - and the CORC team at the time of writing (including E.N): Julian Edbrooke-Childs, Benjamin Richie, Kate Dalzell, Jenna Jacob, Jenny Bloxham, Victoria Zamperoni, Carin Eisenstein, Meera Patel, Andy Whale, Alison Ford, Sally Marriott, Lee Atkins, Danielle Antha, Rebecca Neale.

Miranda Wolpert was (in part) supported by the National Institute for Health Research (NIHR) Collaboration for Leadership in Applied Health Research and Care (CLAHRC) North Thames at Bart's Health NHS Trust. The views expressed are those of the author(s) and not necessarily those of the NHS, the NIHR or the Department of Health and Social Care.

\section{Author Contributions}

Eoin McElroy helped conceptualise the study, conducted all the analyses and drafted the article. Praveetha Patalay conceptualised the study, drafted the article and provided input at all stages of analysis and drafting. Elisa Napoleone prepared the initial dataset, helped conduct the analysis and provided input at all stages of drafting. Miranda Wolpert helped conceptualise the study, reviewed and provided input during drafting. All authors signed off the final draft.

\section{Appendix A. Supplementary data}

Supplementary data to this article can be found online at https://doi. org/10.1016/j.eclinm.2019.02.009.

\section{References}

[1] Kessler RC, Berglund P, Demler O, Jin R, Merikangas KR, Walters EE. Lifetime prevalence and age-of-onset distributions of DSM-IV disorders in the National Comorbidity Survey Replication. Arch Gen Psychiatry 2005;62(6):593-602.

[2] Merikangas KR, He J-p, Burstein M, et al. Lifetime prevalence of mental disorders in US adolescents: results from the National Comorbidity Survey ReplicationAdolescent Supplement (NCS-A). J Am Acad Child Adolesc Psychiatry 2010;49 (10):980-9.

[3] Ferrari AJ, Charlson FJ, Norman RE, et al. Burden of depressive disorders by country, sex, age, and year: findings from the global burden of disease study 2010. PLoS Med 2013;10(11):e1001547.

[4] Kessler RC, Bromet EJ. The epidemiology of depression across cultures. Annu Rev Public Health 2013;34:119-38.

[5] Weersing VR, Weisz JR. Community clinic treatment of depressed youth: benchmarking usual care against CBT clinical trials. J Consult Clin Psychol 2002;70 (2):299.

[6] Fried EI, Nesse RM. Depression sum-scores don't add up: why analyzing specific depression symptoms is essential. BMC Med 2015;13(1):72.

[7] Forbes MK, Wright AG, Markon KE, Krueger RF. Further evidence that psychopathology networks have limited replicability and utility: response to Borsboom et al. (2017) and Steinley et al.(2017). 2017.

[8] Borsboom D, Fried EI, Epskamp S, et al. False alarm? A comprehensive reanalysis of "Evidence that psychopathology symptom networks have limited replicability" by Forbes, Wright, Markon, and Krueger (2017); 2017.

[9] Wichers M, Wigman JT, Bringmann LF, de Jonge P. Mental disorders as networks: some cautionary reflections on a promising approach. Soc Psychiatry Psychiatr Epidemiol 2017;52(2):143-5.

[10] van Borkulo C, Boschloo L, Borsboom D, Penninx BW, Waldorp LJ, Schoevers RA. Association of symptom network structure with the course of depression. JAMA Psychiat 2015;72(12):1219-26.

[11] Schweren L, van Borkulo C, Fried E, Goodyear I. Assessment of symptom network density as a prognostic marker of treatment response in adolescent depression JAMA Psychiat 2017. 
[12] Jacobson NS, Truax P. Clinical significance: a statistical approach to defining meaningful change in psychotherapy research. J Consult Clin Psychol 1991;59(1):12.

[13] Evans C, Margison F, Barkham M. The contribution of reliable and clinically significant change methods to evidence-based mental health. Evid Based Ment Health 1998;1(3):70-2.

[14] Bos FM, Fried EI, Hollon SD, et al. Cross-sectional networks of depressive symptoms before and after antidepressant medication treatment. Soc Psychiatry Psychiatr Epidemiol 2018:1-11.

[15] Fried EI, van Borkulo CD, Epskamp S, Schoevers RA, Tuerlinckx F, Borsboom D. Measuring depression over time... Or not? Lack of unidimensionality and longitudinal measurement invariance in four common rating scales of depression. Psychol Assess 2016;28(11):1354.

[16] Wolpert M, Jacob J, Napoleone E, Whale A, Calderon A, Edbrooke-Childs J. Child-and parent-reported outcomes and experience from child and young People's mental health services 2011-2015. London: CAMHS press; 2016.

[17] Chorpita BF, Moffitt CE, Gray J. Psychometric properties of the revised child anxiety and depression scale in a clinical sample. Behav Res Ther 2005;43(3):309-22.

[18] Epskamp S, Cramer AO, Waldorp LJ, Schmittmann VD, Borsboom D. qgraph: network visualizations of relationships in psychometric data. Journal of Statistical Software 2012;48(4):1-18.

[19] Epskamp S, Borsboom D, Fried EI. Estimating psychological networks and their accuracy: a tutorial paper. Behav Res Methods 2017:1-18.

[20] Opsahl T, Agneessens F, Skvoretz J. Node centrality in weighted networks: generalizing degree and shortest paths. Social networks 2010;32(3):245-51.

[21] van Borkulo C, Boschloo L, Kossakowski J, et al. Comparing network structures on three aspects: A permutation test. Manuscript submitted for publication; 2016. p. 24.

[22] Benjamini Y, Hochberg Y. Controlling the false discovery rate: a practical and powerful approach to multiple testing. I R Stat Soc B Methodol 1995:289-300.

[23] Hiller W, Schindler AC, Lambert MJ. Defining response and remission in psychotherapy research: a comparison of the $\mathrm{RCI}$ and the method of percent improvement. Psychother Res 2012;22(1):1-11.
[24] Rosenbaum PR, Rubin DB. Constructing a control group using multivariate matched sampling methods that incorporate the propensity score. The American Statistician 1985;39(1):33-8.

[25] Ho DE, Imai K, King G, Stuart EA. Matchit: nonparametric preprocessing for parametric causal inference. Journal of Statistical Software 2011;42(8):1-28.

[26] Beard C, Millner A, Forgeard M, et al. Network analysis of depression and anxiety symptom relationships in a psychiatric sample. Psychol Med 2016;46(16):3359-69.

[27] Borsboom D, Cramer AO. Network analysis: an integrative approach to the structure of psychopathology. Annu Rev Clin Psychol 2013;9:91-121.

[28] McGorry PD, Hickie IB, Yung AR, Pantelis C, Jackson HJ. Clinical staging of psychiatric disorders: a heuristic framework for choosing earlier, safer and more effective interventions. Australian and New Zealand Journal of Psychiatry 2006;40(8):616-22.

[29] Wigman JT, van Os J, Thiery E, et al. Psychiatric diagnosis revisited: towards a system of staging and profiling combining nomothetic and idiographic parameters of momentary mental states. PLoS One 2013;8(3):e59559.

[30] Terluin B, de Boer MR, de Vet HC. Differences in connection strength between mental symptoms might be explained by differences in variance: reanalysis of network data did not confirm staging. PloS one 2016;11(11):e0155205.

[31] Kroeze R, Van Veen D, Servaas MN, et al. Personalized feedback on symptom dynamics of psychopathology: a proof-of-principle study. J Person-Orient Res; 2016.

[32] Fried EI, Epskamp S, Nesse RM, Tuerlinckx F, Borsboom D. What are'good'depression symptoms? Comparing the centrality of DSM and non-DSM symptoms of depression in a network analysis. J Affect Disord 2016;189:314-20.

[33] Babadi B, Baek JH, Nierenberg A. 388-a complex networks approach to the symptomatology of mood disorders. Biol Psychiatry 2017;81(10):S158-9.

[34] Fried EI, van Borkulo CD, Cramer AO, Boschloo L, Schoevers RA, Borsboom D. Mental disorders as networks of problems: a review of recent insights. Soc Psychiatry Psychiatr Epidemiol 2017;52(1):1-10. 